\title{
Characterization of Mycobacterium Tuberculosis Complex Strains: A Multicenter Retrospective Greek Study
}

\begin{abstract}
Lamprini Gkaravela $^{1 *}$, Zoi Florou ${ }^{2 *}$, Matthaios Papadimitriou-Olivgeris ${ }^{3}$, Fanourios Kontos ${ }^{4}$, Antigoni Foka1, Afroditi Vasdeki ${ }^{2}$, Fevronia Kolonitsiou ${ }^{1}$, Nikolaos Charokopos ${ }^{5}$, Kiriakos Karkoulias ${ }^{6}$, Markos Marangos $^{3}$, Evangelos D Anastassiou ${ }^{1}$, Loukia Zerva ${ }^{4}$, Efthimia Petinaki ${ }^{2}$ and Iris Spiliopoulou ${ }^{1}$
\end{abstract}

${ }^{1}$ Department of Microbiology, School of Medicine, Greece

${ }^{2}$ Department of Microbiology, School of Medicine, Greece

${ }^{3}$ Division of Infectious Diseases, School of Medicine, Greece

${ }^{4}$ Laboratory of Clinical Microbiology, Greece

${ }^{5}$ Department of Pulmonology, Greece

${ }^{6}$ Department of Pulmonology, Greece

*Both authors contributed equally to this study

*Corresponding author: Iris Spiliopoulou, MD, PhD, Department of Microbiology, School of Medicine University of Patras, Patras 26504, Greece

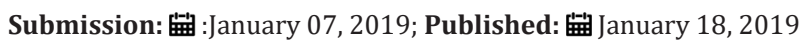

\begin{abstract}
The aim was to compare clinical characteristics, antimicrobial resistance and 24-loci variable numbers of tandem repeats of mycobacterial interspersed repetitive units (MIRU-VNTR) patterns among M. tuberculosis complex isolates (MTBC) recovered from patients living in Greece during a two-year period (2009-10). Isolates from 117 tuberculosis patients identified as MTBC by a molecular method were phenotypically tested for their antimicrobial susceptibility. Clonality was accessed by MIRU-VNTR. Among 117 patients with confirmed microbiologic tuberculosis, 90 (76.9\%) were of Greek origin, and 27 (23.1\%) foreigners. Only 67 out of the 117 (57.3\%) patients were officially reported to the Hellenic Center for Disease Control and Prevention. Phenotypic susceptibility testing revealed 10 isolates resistant to isoniazid (8.5\%), two to rifampicin (1.7\%), five to ethambutol (4.3\%) and 17 to streptomycin (14.5\%). In total two multidrug-resistant strains (1.7\%) were detected. MIRU-VNTR classified 35 strains (29.9\%) into 10 Families, whereas, 82 (70.1\%) were not classified into any known Family. MTBC strains belonging to known Families were more commonly isolated from people living in cities and foreigners and showed a trend to higher resistance percentages to isoniazid and rifampicin. Most isolates from Greek rural areas do not belong to any known Family and are characterized by lower resistance rates.
\end{abstract}

Keywords: MIRU-VNTR; Tuberculosis; Clones; resistance; Immigration

\section{Introduction}

Even though tuberculosis incidence has steadily decreased during the last three decades, worldwide, there is a great disparity among different areas. Countries of the African and South-East Asia Regions record the highest rates, whereas, countries of the European and American Regions exhibit the lowest (254 and 240 vs 32 and 27 cases per 100000 habitants, respectively) [1]. This fact poses an immediate threat to tuberculosis control programs in low incidence countries when large populations from areas with high incidence migrate to low incidence ones. In most European countries, new tuberculosis cases are reported among foreigners rather than in native population [2]. This is also similar for Greece, where the incidence among native population has fallen during the last decade, while, the incidence among foreigners steadily increases [2].
Another important issue is the emergence and worldwide dissemination of multi-drug resistant (MDR) Mycobacterium tuberculosis isolates [1]. Areas with high rates of MDR are the East and Central Asia (reaching 27\% among new cases) and countries of the former Soviet Union (38\%) [1]. These high rates have been partially attributed to clonal dissemination of strains belonging to the Beijing family, to which a considerable number of MDR is assigned [37]. The Beijing family originated in the area of China, approximately 6600 years ago and is successfully disseminated worldwide in several waves coinciding with migration of large populations $[7,8]$. Nowadays, it remains endemic in Central Asia and Russia [7,8]. The dissemination of MDR strains following large human population migration is of utmost importance, since during the last year 
approximately one million refugees from the area of Middle East (Syrian Arab Republic, Iraq) and Central Asia (Afghanistan, Pakistan) have migrated through Mediterranean countries to Europe due to armed conflicts in their homelands [9]. In one Greek study including only drug resistant cases, the authors showed that most isolates (28 out of 30) from patients born in Greece belonged to known families. More specifically, 12 strains belonged to Haarlem, eight to Beijing, followed by T2-Uganda $(n=4)$, LAM ( $n=3)$, Cameroon $(n=2)$, Delhi/CAS (n=1). Two strains were not classified into any known Family [10].

The aim of the present study was to compare clinical characteristics, antimicrobial resistance and 24-loci variable numbers of tandem repeats of mycobacterial interspersed repetitive units (MIRU-VNTR) patterns among $M$. tuberculosis complex isolates recovered from patients living in different parts of Greece.

\section{Material and Methods}

\section{Setting}

A total of 117 M. tuberculosis complex (MTBC) clinical isolates (one per patient) were collected from two participating tertiary care hospitals during a two year period (2009-10); 62 isolates from the Hospital A and 55 from the Hospital B. These hospitals receive patients and samples from surrounding regions comprising about one fifth of the country's population (two million). Isolates were recovered under routine diagnostic procedures for tuberculosis.

Patients' chart reviews were used in order to collect epidemiologic data. Parameters assessed included demographic characteristics (age, sex), residency, country of birth, immunosuppression (including HIV infection) and site of infection. Data concerning the declaration or not of the case to the Hellenic Center for Disease Control and Prevention (KEELPNO) was also collected (http:// www.keelpno.gr/)

Mycobacteria were identified to species level by a reverse line blot hybridization (RLBH) assay (GenoType Mycobacterium CM; Hain Lifescience GmbH, Nehren, Germany). MTBC strains were isolated from sputa $(n=58)$, bronchoalveolar lavages $(n=23)$, gastric aspirates $(n=10)$, tissue specimens $(n=8)$, synovial fluids $(n=8)$, pus $(n=5)$, urine $(n=2)$, blood $(n=2)$ and cerebrospinal fluid $(n=1)$

\section{Antimicrobial susceptibility testing}

Susceptibility testing was performed in all isolates by a phenotypic method BACTEC Mycobacteria Growth Indicator Tube (MGIT) 960 SIRE kit (MGIT-SIRE, Becton Dickinson, Le Pont-DeClaix, France) for streptomycin (STR), isoniazid (INH), rifampicin (RMP) and ethambutol (EMB) according to the manufacturers' instructions. Strains resistant to INH and RMP were characterized as multi-drug resistant (MDR) [2].

\section{Genotyping}

For genotypic analysis only the first isolate obtained from each patient was examined. DNA extraction was performed from bacterial colonies using QIAamp DNA Mini Kit (Qiagen GmbH, Hilden, Ger- many). All isolates were genotyped by the MIRU-VNTR method by analyzing 24 polymorphic loci [11]. The corresponding lineage of each strain was then determined by the use of MIRU-VNTRplus database (http://miru-vntrplus.org) according to published instructions [12]. When two or more isolates were identical by MIRU-VNTR, they were classified as clustered.

\section{Statistical analysis}

Statistical analyses of demographic, laboratory and clinical data (obtained from patients' chart reviews and/or notification forms) as well as genotyping data were performed with SPSS version 19.0 (SPSS, Chicago, IL, USA) software. Continuous variables were assessed with the Mann-Whitney U-test and categorical variables were analyzed by using Fisher's exact test or the Chi-squared test, as appropriate.

\section{Results}

\section{Isolates and resistance patterns}

In total, 117 patients with confirmed microbiologic and clinical tuberculosis were included. Antimicrobial susceptibility testing by a phenotypic method was applied in all 117 isolates and revealed $10(8.5 \%)$ resistant to INH, two $(1.7 \%)$ to RMP, five (4.3\%) to EMB and $17(14.5 \%)$ to STR. Two MDR strains were detected (1.7\%).

\section{MIRU-VNTR}

MIRU-VNTR typing was performed in all isolates. Thirty-five (29.9\%) were classified into 10 Families; Haarlem (14 isolates, 12.0\%), Latin American-Mediterranean (LAM; six, 5.1\%), S clade (five, 4.3\%), Delhi/CAS (two, 1.7\%), Beijing (two, 1.7\%), Turkey (TUR; two, 1.7\%), East African-Indian (EAI; one, 0.9\%), Ugandal (one, 0.9\%), Ural (one, 0.9\%), and X (one, 0.9\%), whereas, 82 $(70.1 \%)$ were not classified into any known Family. In total one cluster was identified comprised by two cases (not classified in known families), but no evident correlation was found. Of two MDR strains, one belonged to TUR (patient originated from Bulgaria) and the other to $\mathrm{X}$ families (Greek patient).

\section{Clinical characteristics}

Among 117 infected patients, 90 (76.9\%) were of Greek origin, whereas, the remaining 27 (23.1\%) were foreigners (seven from Bulgaria, five from Romania, four from Albania, two from Pakistan, two from India, and one from each Afghanistan, Cyprus, Georgia, Indonesia, Philippines, Poland, and USA). No statistical difference was observed for all parameters among Greeks and foreigners between the participating hospitals. Only 67 patients (57.3\%) were officially reported to Hellenic Center for Disease Control and Prevention (KEELPNO). No registered address was found for 14 out of 117 patients.

Foreigners as compared to Greek patients were younger, prone to infection by MTBC isolates belonging to known Families, lived in cities (>100000 inhabitants) and demonstrated more frequently lymph node involvement. The comparison of clinical characteristics among Greek and Foreign patients are shown in Table 1. 
Table 1: Demographic and clinical characteristics of tuberculosis between Greek and Foreigner patients living in the country.

\begin{tabular}{|c|c|c|c|}
\hline Characteristics & Greeks (90) & Foreigners (27) & $\mathbf{P}$ \\
\hline Male sex & $59(65.6 \%)$ & $22(81.5 \%)$ & 0.155 \\
\hline Age (years \pm SD) & $59.7 \pm 22.8$ & $41.4 \pm 17.3$ & $<0.001$ \\
\hline \multicolumn{4}{|l|}{ Residency $^{\mathrm{a}}$} \\
\hline Cities (>100000 inhabitants) & $12(14.6 \%)$ & $8(38.1 \%)$ & 0.027 \\
\hline Large towns (20000-100000 inhabitants) & $38(46.3 \%)$ & $5(23.8 \%)$ & \\
\hline Towns and villages (<20000 inhabitants) & $32(39.0 \%)$ & $8(38.1 \%)$ & \\
\hline HIV carrier & $2(2.2 \%)$ & $3(11.1 \%)$ & 0.08 \\
\hline Immunosuppression (other than HIV) & $4(8.5 \%)$ & $3(15.0 \%)$ & 0.418 \\
\hline Pulmonary disease & $75(83.3 \%)$ & $20(74.1 \%)$ & 0.276 \\
\hline Extra-pulmonary disease & $25(27.8 \%)$ & $11(40.7 \%)$ & 0.237 \\
\hline Pleural effusion & $7(7.8 \%)$ & $1(3.7 \%)$ & 0.68 \\
\hline Lymph node involvement & $7(7.8 \%)$ & $7(25.9 \%)$ & 0.018 \\
\hline Gastrointestinal involvement & $2(2.2 \%)$ & $1(3.7 \%)$ & 0.548 \\
\hline Osteoarticular involvement & $1(1.1 \%)$ & $1(3.7 \%)$ & 0.41 \\
\hline Urogenital involvement & $3(3.3 \%)$ & $1(3.7 \%)$ & 1.00 \\
\hline Skin involvement & $3(3.3 \%)$ & $1(3.7 \%)$ & 1.00 \\
\hline Central nervous system involvement & $1(1.1 \%)$ & $1(3.7 \%)$ & 0.41 \\
\hline Pulmonary and extra-pulmonary disease & $12(13.3 \%)$ & $5(18.5 \%)$ & 0.538 \\
\hline Classified in Families & $21(23.3 \%)$ & $14(51.9 \%)$ & 0.008 \\
\hline \multicolumn{4}{|l|}{ Antimicrobial resistance } \\
\hline INH & $7(7.8 \%)$ & $3(11.1 \%)$ & 0.695 \\
\hline RMP & $1(1.1 \%)$ & $1(3.7 \%)$ & 1.00 \\
\hline MDR & $1(1.1 \%)$ & $1(3.7 \%)$ & 1.00 \\
\hline EMB & $2(2.2 \%)$ & $3(11.1 \%)$ & 0.08 \\
\hline STR & $13(14.4 \%)$ & $4(14.8 \%)$ & 1.00 \\
\hline
\end{tabular}

MIRU-VNTR: 24-loci variable numbers of tandem repeats of mycobacterial interspersed repetitive units; SD: standard deviation; HIV: human immunodeficiency virus; INH; isoniazid; RMP: rifampicin; MDR: multidrug-resistant; EMB: ethambutol; STR: streptomycin

${ }^{a}$ Data were available for 103 patients.

${ }^{b}$ Data were available for 67 patients.

Demographic and clinical characteristics of patients according plex strains belonging to known Families were more commonly isoto MIRU-VNTR results are depicted in Table 2. M. tuberculosis com- lated from people living in cities and foreigners.

Table 2: Demographic and clinical characteristics of tuberculosis patients according to M. tuberculosis complex strains' lineage determination by MIRU-VNTR.

\begin{tabular}{|c|c|c|c|}
\hline Characteristics & Classified into Families (35) & Unclassified (82) & $\mathrm{P}$ \\
\hline Male sex & $25(71.4 \%)$ & $56(68.3 \%)$ & 0.829 \\
\hline Age (years \pm SD) & $48.6 \pm 23.9$ & $58.0 \pm 21.9$ & 0.064 \\
\hline \multicolumn{4}{|l|}{ Residency $^{\mathrm{a}}$} \\
\hline Cities (>100000 inhabitants) & $13(44.8 \%)$ & $7(9.5 \%)$ & $<0.001$ \\
\hline Large towns (20000-100000 inhabitants) & $8(27.6 \%)$ & $35(47.3 \%)$ & \\
\hline Towns and villages ( $<20000$ inhabitants) & $8(27.6 \%)$ & $32(43.2 \%)$ & \\
\hline HIV carrier & $2(5.7 \%)$ & $3(3.7 \%)$ & 0.635 \\
\hline Immunosuppression (other than HIV) ${ }^{\mathrm{b}}$ & $3(12.5 \%)$ & $4(9.3 \%)$ & 0.695 \\
\hline
\end{tabular}




\begin{tabular}{|c|c|c|c|}
\hline Foreigners & $14(40.0 \%)$ & $13(15.9 \%)$ & 0.008 \\
\hline Pulmonary disease & $29(82.9 \%)$ & $66(80.5 \%)$ & 1.00 \\
\hline Extra-pulmonary disease & $10(28.6 \%)$ & $26(31.7 \%)$ & 0.829 \\
\hline Pleural effusion & $1(2.9 \%)$ & $7(8.5 \%)$ & 0.433 \\
\hline Lymph node involvement & $5(14.3 \%)$ & $9(11.9 \%)$ & 0.756 \\
\hline Gastrointestinal involvement & $0(0.0 \%)$ & $3(3.7 \%)$ & 0.553 \\
\hline Osteoarticular involvement & $1(2.9 \%)$ & $1(1.2 \%)$ & 0.511 \\
\hline Urogenital involvement & $1(2.9 \%)$ & $3(3.7 \%)$ & 1.00 \\
\hline Skin involvement & $2(5.7 \%)$ & $2(2.4 \%)$ & 0.582 \\
\hline Central nervous system involvement & $0(0.0 \%)$ & $2(2.4 \%)$ & 1.00 \\
\hline Pulmonary and extra-pulmonary disease & $5(14.3 \%)$ & $12(14.6 \%)$ & 1.00 \\
\hline \multicolumn{4}{|l|}{ Antimicrobial resistance } \\
\hline INH & $6(17.1 \%)$ & $4(4.9 \%)$ & 0.063 \\
\hline RMP & $2(5.7 \%)$ & $0(0.0 \%)$ & 0.088 \\
\hline MDR & $2(5.7 \%)$ & $0(0.0 \%)$ & 0.088 \\
\hline EMB & 3 (8.6\%) & $2(2.4 \%)$ & 0.157 \\
\hline STR & $7(20.0 \%)$ & $10(12.2 \%)$ & 0.269 \\
\hline
\end{tabular}

MIRU-VNTR: 24-loci variable numbers of tandem repeats of mycobacterial interspersed repetitive units; SD: standard deviation; HIV: human immunodeficiency virus; INH; isoniazid; RMP: rifampicin; MDR: multidrug-resistant; EMB: ethambutol; STR: streptomycin

aData were available for 103 patients.

${ }^{\mathrm{b}}$ Data were available for 67 patients.

\section{Discussion}

A very important and unexpected finding of the present study is the underreporting of tuberculosis in Greece, since only $57.3 \%$ of microbiologically confirmed cases were actually reported to KEELPNO. This may provoke serious repercussions in public health, since if a case is not notified to authorities no contact tracing is implemented. This issue was also addressed in a previous Greek study that used anti-tuberculosis drug consumption from 2004-8, where underreporting was approximately $80 \%$ corresponding to an incidence of five times higher than the official notification rate [13]. Since underreporting with the present applicable procedures remains high, a need for an alternative notification system, which may be based on anti-tuberculosis drug consumption, is imperative $[14,15]$. Strains from the present study belonged to multiple Families and most of them (70.1\%) were not classified into any known Family. The percentage of unclassified strains is higher as compared to previous studies from Europe where it was less than $13 \%$. This finding is reinforced by the fact that foreigners from our study have higher percentage of infection by strains classified in known Families, as compared to Greeks [15-17]. The percentage of foreigners in our study is lower than that formally reported by the ECDC [2]. We found that patients living in cities (>100000 inhabitants) are more frequently infected with strains belonging to known Families as compared to other regions of Greece, pointing to a different epidemiology between the high density urban population and rural areas. Strains belonging to known Families were more commonly resistant to INH and RMP, probably due to the fact that they are associated with foreigners originating from countries with higher percentage of resistance [2]. This was also shown in a previous Greek study from "Sotiria" Hospital, in Athens, where 93.3\% of drug resistant MTBC strains belonged to Known Families, including 12 in Haarlem and eight in Beijing [10]. The percentage of pulmonary tuberculosis was similar between patients infected by strains belonging to known Families or unclassified ones. Even though no difference of rates of extrapulmonary tuberculosis was observed, lymphadenitis was more common among patients infected by strains belonging in Known Families. Moreover, the percentage of pulmonary tuberculosis was comparable to that reported by ECDC concerning Greece, but the rate of extrapulmonary disease was significantly higher. This may be explained by the fact that extrapulmonary tuberculosis due to its difficult differential diagnosis is not formally reported at the Greek authorities by the clinicians, as pulmonary cases [2].

Resistance of MTBC isolates to INH (8.5\%) was comparable to that reported in a previous Greek study regarding the period 2005-9 (6.5\%), while, resistance to RMP is lower in the present one $(1.7 \%$ vs $3.9 \%$ ) [18]. The percentage of MDR isolates in this study $(1.7 \%)$ is also lower as compared to previous reports $(3.0 \%)$ and ECDC data (4.3\%) $[2,18]$. As previously shown, resistance rates to INH or RMP of MTBC isolates recovered from foreigners is higher than the native population, underlying the importance of screening and appropriate treatment of latent and active tuberculosis among immigrants [18-20]. 
Strains belonging to Beijing family are usually MDR. This was also shown by a previous Greek study where 10 out of 18 strains (55.6\%) were MDR. More specifically, most of them were recovered from immigrants from Russia and countries of Central Asia, where Beijing strains predominate [10]. However, none of the Beijing isolates in the present study were MDR. This is due to the fact that most foreigners $(82.9 \%)$ originated from countries where Beijing is seldom identified [7]. Moreover, the countries of foreigners' participating in this study reflect the flux of immigration towards Greece before 2010 (mainly Balkan countries or those of the former Soviet Union). This has changed abruptly the last three years, after the armed conflict in Syrian Arab Republic and Iraq, and the high influx of refugees in Greece from Middle East and Central Asia [9]. In total, till the end of December 2017, more than a million refugees have arrived in Greece, mainly from Syrian Arab Republic (47\%), Afghanistan (24\%), Iraq (15\%), and Pakistan (5\%), areas with higher incidence of tuberculosis (Afghanistan: 189; Iraq: 43; Pakistan: 270; Syrian Arab Republic: 17 per 100000) than Greece (4.8 per 100000) [9].

Aforementioned countries also exhibit higher percentages of multi-drug resistant tuberculosis among new cases and relapses (Afghanistan: 4\% in total; Iraq: 1\% and 20\%; Pakistan: 3.7\% and 18\%; Syrian Arab Republic: 6\% and 31\%, respectively) as compared to Greece (1\% and 9\%), due to introduction of the Beijing Family in Central Asia and Middle-East [1,7]. The immigration influx in countries with low rates of resistance may pose serious problems in their public health systems due to transmission of multidrug strains in the community, as previously shown $[20,21]$. It was theorized that strains of the Beijing Family have successfully disseminated worldwide by exploiting humanity's immigration pathways $[7,8]$. This does not mean that introduction of strains belonging to the Beijing Family in European countries will always result in its dissemination and subsequent rise of resistance rates. This is shown in a study from Sweden, which neighbor countries with high prevalence of the Beijing Family (Russia, Baltic countries); however, the incidence of Beijing isolates did not rise in Sweden during 1994-2008 [22].

This study has some limitations. It is a retrospective analysis and for some cases insufficient data were available from patients' charts. Moreover, we did not perform Spoligotyping or Whole Genome Sequencing among unclassified strains to identify if they belonged to any known Family or if they are indeed unclassified.

To conclude, M. tuberculosis complex strains isolated from a large part of Greek population belonged to several Families, most of which were not classified to known ones, and are associated with low rates of resistance. Isolates from foreigners were associated with higher percentages of resistance to INH and RMP, suggesting that the recent immigration influx from areas with high incidence of resistance and presence of the Beijing Family, such as Middle East and Central Asia, to Europe, will put to the test our tuberculosis control programs.

\section{Acknowledgements}

A part of this work was presented as a Poster presentation at the 26th European Congress of Clinical Microbiology and Infectious Diseases, 9-12 April 2016, Amsterdam, Netherlands.

Funding: This research did not receive any specific grant from funding agencies in the public, commercial, or not-for-profit sectors.

\section{Ethical standards}

Bioethics Committees of the UGHP and UGHL approved the study and waived the need for informed consent (Approval Numbers 317 and 3821, respectively).

\section{References}

1. World Health Organization (WHO) (2017) Global tuberculosis report.

2. European Centre for Disease Prevention and Control/WHO Regional Office for Europe (2017) Tuberculosis surveillance and monitoring in Europe, 2018. Stockholm, Europe.

3. Zhao LL, Chen Y, Chen ZN, Liu HC, Hu PL, et al. (2014) Prevalence and molecular characteristics of drug-resistant Mycobacterium tuberculosis in Hunan, China. Antimicrob Agents Chemother 58(6): 3475-3480.

4. Mokrousov I, Vyazovaya A, Otten T, Zhuravlev V, Pavlova E, et al. (2012) Mycobacterium tuberculosis population in northwestern Russia: an update from Russian-EU/Latvian border region. Plos One 7(7): e41318.

5. Zhang Z, Lu J, Liu M, Wang Y, Qu G, et al. (2015) Genotyping and molecular characteristics of multidrug-resistant Mycobacterium tuberculosis isolates from China. J Infect 70(4): 335-345.

6. Lipin MY, Stepanshina VN, Shemyakin IG, Shinnick TM (2007) Association of specific mutations in katG, rpoB, rpsL and rrs genes with spoligotypes of multidrug-resistant Mycobacterium tuberculosis isolates in Russia. Clin Microbiol Infect 13(6): 620-626.

7. Couvin D, Rastogi N (2015) Tuberculosis - A global emergency: Tools and methods to monitor, understand, and control the epidemic with specific example of the Beijing lineage. Tuberculosis (Edinb) 95 Suppl 1: S177-S189.

8. Merker M, Blin C, Mona S, Duforet FN, Lecher S, et al. (2015) Evolutionary history and global spread of the Mycobacterium tuberculosis Beijing lineage. Nat Genet 47(3): 242-249.

9. United Nations High Commissioner for Refugees (UNHCR) (2017) Greece Sea arrivals dashboard - December 2017. UNHCR.

10. Rovina N, Karabela S, Constantoulakis P, Michou V, Konstantinou K, et al. (2011) MIRU-VNTR typing of drug-resistant tuberculosis isolates in Greece. Ther Adv Respir Dis 5(4): 229-236.

11. Supply P, Allix C, Lesjean S, Cardoso OM, Rusch GS, et al. (2006) Proposal for standardization of optimized mycobacterial interspersed repetitive unit-variable-number tandem repeat typing of Mycobacterium tuberculosis. J Clin Microbiol 44(12): 4498-4510.

12. Allix BC, Harmsen D, Weniger T, Supply P, Niemann S (2008) Evaluation and strategy for use of MIRU-VNTR plus, a multifunctional database for online analysis of genotyping data and phylogenetic identification of Mycobacterium tuberculosis complex isolates. J Clin Microbiol 46(8): 2692-2699.

13. Lytras T, Spala G, Bonovas S, Panagiotopoulos T (2012) Evaluation of tuberculosis underreporting in Greece through comparison with antituberculosis drug consumption. Plos One 7(11): e50033. 
14. Jelastopulu E, Alexopoulos EC, Venieri D, Tsiros G, Komninou G, et al (2009) Substantial underreporting of tuberculosis in West Greece: implications for local and national surveillance. Euro Surveill 14(11): 19152.

15. Bonura C, Gomgnimbou MK, Refregier G, Aleo A, Fasciana T, et al. (2014) Molecular epidemiology of tuberculosis in Sicily, Italy: what has changed after a decade? BMC Infect Dis 14: 602 .

16. Smit PW, Haanpera M, Rantala P, Couvin D, Lyytikainen O, et al. (2013) Molecular epidemiology of tuberculosis in Finland, 2008-2011. Plos One 8(12): e85027.

17. Svensson E, Millet J, Lindqvist A, Olsson M, Ridell M, et al. (2011) Impact of immigration on tuberculosis epidemiology in a low-incidence country. Clin Microbiol Infect 17(6): 881-887.

18. Papaventsis D, Nikolaou S, Karabela S, Ioannidis P, Konstantinidou E et al. (2010) Tuberculosis in Greece: bacteriologically confirmed cases and anti-tuberculosis drug resistance, 1995-2009. Euro Surveill 15(28): 19614.
19. Calderon HR, Ruiz GJM, Cabello CN, Canora LJ (2016) Tuberculosis and immigration in an area of southwest Madrid. Int J Tuberc Lung Dis 20(4): 530-535.

20. Lombardi G, Dal MP, Denicolo A, Tadolini M, Martelli G, et al. (2014) Trend of microbiologically-confirmed tuberculosis in a low-incidence setting with high immigration rates. BMC Public Health 14: 340 .

21. Fortun J, Martin DP, Navas E, Lopez VR, Pintado V, et al. (2011) Changes in the epidemiology of tuberculosis: the influence of international migration flows. Enferm Infecc Microbiol Clin 29(9): 654-659.

22. Ghebremichael S, Groenheit R, Pennhag A, Koivula T, Andersson E, et al. (2010) Drug resistant Mycobacterium tuberculosis of the Beijing genotype does not spread in Sweden. PloS One 5(5): e10893.
Creative Commons Attribution 4.0 International License

For possible submissions Click Here

\section{Submit Article}

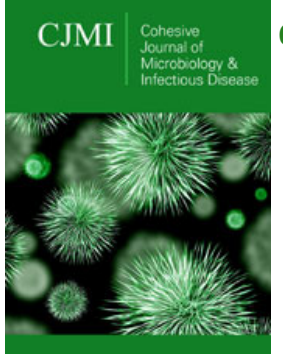

Cohesive Journal of Microbiology \& Infectious Disease

\section{Benefits of Publishing with us}

- High-level peer review and editorial services

- Freely accessible online immediately upon publication

- Authors retain the copyright to their work

- Licensing it under a Creative Commons license

- Visibility through different online platforms 